\title{
The maintenance and exploitation of plant genetic resources - state of the art
}

\author{
Börner A. ${ }^{1 *}$, Nagel M. ${ }^{1}$, Agacka-Mołdoch M. ${ }^{1,2}$, Rehman Arif M.A. ${ }^{1,3}$, Lohwasser U. ${ }^{1}$, \\ Riewe D. ${ }^{1,4}$, Wiebach J. ${ }^{1,5}$, Altmann T. ${ }^{1}$, Pshenichnikova T.A. ${ }^{6}$, Khlestkina E. ${ }^{7}$ \\ ${ }^{1}$ Leibniz Institute of Plant Genetics and Crop Plant Research (IPK), Gatersleben, Germany \\ ${ }^{2}$ Institute of Soil Science and Plant Cultivation, Puławy, Poland \\ ${ }^{3}$ Nuclear Institute of Agriculture and Biology, Faisalabad, Pakistan \\ ${ }^{4}$ Julius Kühn-Institute, Federal Research Centre for Cultivated Plants, Institute for Ecological Chemistry, \\ Plant Analysis and Stored Product Protection, Berlin, Germany \\ ${ }^{5}$ Charité - Universitätsmedizin Berlin, corporate member of Freie Universität Berlin, \\ Humboldt-Universität zu Berlin, and Berlin Institute of Health, Institute of Biometry \\ and Clinical Epidemiology, Berlin, Germany \\ ${ }^{6}$ Institute of Cytology and Genetics, SB RAS, Novosibirsk, Russia \\ ${ }^{7}$ N.I. Vavilov All-Russian Institute of Plant Genetic Resources (VIR), St. Petersburg, Russia \\ *e-mail: boerner@ipk-gatersleben.de
}

There are two main strategies for maintaining plant genetic resources: ex situ and in situ conservation. Ex situ conservation means the conservation of the biological diversity outside their natural habitats. In situ conservation means the conservation of ecosystems, natural habitats and viable populations of species in their natural surroundings. Ex situ conservation is the most significant and widespread mean of conserving plant genetic resources. Mainly, accessions are maintained in specialized facilities known as genebanks. World-wide 7.4 million accessions are stored in about 1,750 ex situ genebanks. Considering major crop groups about 45 percent of all the accessions in the world's genebanks are cereals, followed by legumes (15 percent), fruits and forage crops (each 6-9 percent) as well as roots and tubers, oil crops and fibre crops (each 2-3 percent). It is estimated that $90 \%$ of all genebank holdings are stored as seeds whereas less than $10 \%$ and less than $1 \%$ are maintained in vivo (field genebanks) and in vitro (tissue culture and cryo preservation), respectively. Clearly, seed storage is the predominant mode of plant genetic resources conservation. With a total inventory of 150,000 accessions from 3,212 plant species and 776 genera, the 'Federal ex situ Genebank of Germany' in Gatersleben holds one of the most comprehensive collections worldwide. It comprises wild and primitive forms, landraces as well as old and more recent cultivars of mainly cereals but also other crops. Since the majority of genebank accessions globally are stored in the form of seed, seed longevity is of particular importance for crop germplasm preservation. At the IPK research was initiated for a range of crops stored in the genebank over decades. Variation between crop species was detected. However, there is also intraspecific variation within genebank collections. It was concluded that the differences in germination after long term storage are genetically based. Therefore, genetic analyses of seed longevity were initiated. Genetic mapping was performed for barley, wheat, oilseed rape and tobacco. In addition, mass spectrometry based untargeted metabolite profiling experiments were performed in order to detect biochemical changes coinciding with loss in seed germination. GC-MS analysis of the polar metabolome of wheat and barley identified glycerol and related intermediates as highly correlated to germination rate. Therefore, the lipidomic composition of a wheat panel was investigated using highresolution liquid chromatography-mass spectrometry (LC-MS). A high proportion of tentative oxidized lipids was detected, suggesting lipid oxidation as the causal trigger for membrane degradation. Beside research on seed storability genebank accessions and genetic stocks have been extensively used for genetic and genomic studies. Data on mapping of loci/marker trait associations for a range of different traits will be presented. 